\title{
Robust stability of hybrid Roesser models against parametric uncertainty: a general approach
}

\author{
Mariem Ghamgui • Nima Yeganefar • \\ Olivier Bachelier · Driss Mehdi
}

Received: 19 April 2012 / Revised: 6 November 2012 / Accepted: 26 November 2012 /

Published online: 14 December 2012

C) Springer Science+Business Media New York 2012

\begin{abstract}
This paper aims at proposing a general framework for the establishement of LMI conditions to analyse the robust stability of a singular hybrid Roesser model subject to parametric uncertainties. The uncertain parameters are involved through implicit Linear Fractional Representations (LFR). Special focus is put on the influence of the number of uncertain parameters and the dimensionality of the model. More precisely it is shown that each dimension can nearly be regarded as an uncertain parameter and the other way around. Therefore, their influence on the conservatism of the obtained condition is very similar.
\end{abstract}

Keywords $n$ D hybrid Roesser models · LMI conditions · Implicit LFR-uncertainty

\section{Introduction}

The past few decades have witnessed a large attention paid to the study of multidimensional ( $n \mathrm{D}$-systems) as highlighted in the reference books Bose (1982), Gałkowski and Wood (2001), Kaczorek (1985). The interest in such systems lies in the wide (and perhaps still unknown) variety of their applications, e.g. $n \mathrm{D}$-filtering (Lu and Antoniou 1992), $n \mathrm{D}$-coding and decoding (Shi and Zhang 2002), image and signal processing (Bracewell 1995; Dudgeon and Merserau 1984), or more recently, repetitive processes (Rogers et al. 2007).

Two basic state-space models, that can be transformed one into the other, are used to describe $n \mathrm{D}$-systems. The first is due to Roesser (1975) and the second one was proposed by Fornasini and Marchesini (1978). In this paper, the attention is focused on the former one and more precisely on an hybrid implicit extension of this model.

As for conventional 1D models, the state-space methods are very popular in the realm of $n \mathrm{D}$-system theory since efficient numerical linear algebra routines can be used to analyse the models. They can lead to stability conditions which are expressed in terms of linear

M. Ghamgui · N. Yeganefar · O. Bachelier $(\varangle) \cdot$ D. Mehdi

University of Poitiers LIAS-ENSIP, Bâtiment B25, 2 rue Pierre Brousse,

B.P. 633, 86022 Poitiers Cedex, France

e-mail: Olivier.Bachelier@univ-poitiers.fr 
matrix inequalities (LMI) (Boyd et al. 1994)—particularly "Lyapunov-like inequalities"that give an interpretation in the sense of Lyapunov, or rather of the existence of a Lyapunov function (Gałkowski et al. 2003; Hinamoto 1993; Kaczorek 1985; 1994; Henrion 2001; Kaczorek 2009). The proposed LMI stability conditions, when tractable, are usually only sufficient. Note that some special cases can be dealt with using non conservative conditions (Ebihara et al. 2006). However, these conditions are only formulated for $2 \mathrm{D}$ discrete systems and cannot be easily extended to control design case.

In fact the amount of contributions proposing "Lyapunov-like conditions" can no longer be estimated. Conditions for the discrete case, the continuous case, the 2D-case, the 3D-case, exist but it becomes quite difficult to make connections between these numerous conditions. The various proofs are based upon matrix manipulation, they often sound like each other but it is nonetheless quite difficult to find a formal link between the different results. This is one of the purpose of this paper. We try to propose a quite general framework to address the stability analysis of $n \mathrm{D}$ hybrid Roesser models. Starting from the well-known (but non tractable) stability conditions based upon characteristic polynomials (Jury 1978; Agathoklis 1988; Piekarski 1977), we relax these conditions using a powerful tool, well-known in the realm of 1D-systems, namely the full block S-procedure. It allows us to establish a sufficient LMI condition for the stability of an $n \mathrm{D}$ hybrid Roesser model which encompasses many results of the literature. The connection with the Lyapunov framework is emphasized.

Moreover, to make the result even more general, we also consider parametric uncertainty of the model. These parameters are involved through rational expressions in the entries of the model matrices. To make our description as general as possible, we consider implicit linear fractional representations (LFRs) which are well adapted to our reasoning and contribute to reduce the conservatism of the obtained LMI conditions for robust stability.

A particular issue in the paper is our discussion concerning the resemblance between the ways that Laplace or advance operators and uncertain parameters are handled and influence the pessimism of the conditions. In that sense, the paper is really addressed to the community of $n D$-systems to explain how some powerful tools which can be quite familiar to people working on robust control, can be efficiently exploited to formulate a generic approach to the robust analysis of uncertain $n \mathrm{D}$ Roesser models.

The paper is organized as follows. Section 2 introduces the implicit hybrid Roesser and particularly insists on the uncertainty description. Section 3 states the main result by showing a quite generic approach to the relaxation of well known stability conditions into tractable LMI conditions. Section 4 focuses on the above-evoked resemblance between uncertain parameters and Laplace (or advance) operators, showing that each of them induce a decision matrix in the LMI system. Conservatism and possible further investigations are also commented. Section 5 proposes an illustrating example before the paper is concluded in Sect. 6.

Notations In the sequel, $\mathbb{R}$ denotes the set of real values whereas $\mathbb{C}$ denotes the set of complex values. The matrix inequalities must be understood in the sense of Löwner i.e. $M>0$ (resp. $M<0, M \geq 0$ and $M \leq 0$ ) means that the Hermitian matrix $M$ is positive definite (resp. negative definite, positive semi-definite or negative semi-definite). The symbol ' $\star$ ' denotes the Redheffer-like product:

$$
\begin{aligned}
\Delta_{M} \star \mathscr{M} & =\Delta_{M} \star\left[\begin{array}{c|c}
A_{M} & B_{M} \\
\hline C_{M} & D_{M} \\
\hline E_{M} & F_{M}
\end{array}\right] \\
& =D_{M}+C_{M}\left(E_{M}-\Delta_{M} A_{M}\right)^{-1}\left(\Delta_{M} B_{M}-F_{M}\right) .
\end{aligned}
$$


For a given subset $\mathscr{S}$ of the set $\mathscr{X}$, the set $\mathscr{X} \backslash \mathscr{S}$ denotes the set of the elements of $\mathscr{X}$ which do not belong to $\mathscr{S}$. Also let us define the following notations:

$$
\bigoplus_{i=1}^{k} M_{i}=\underset{i=1, \ldots k}{\operatorname{diag}}\left\{M_{i}\right\}
$$

At last, $(\bullet)^{\prime}$ denotes an evaded part, block or factor in an expression that can be recovered by invoking symmetry.

\section{Uncertain Roesser model}

One of the most commonly used model for $n$-D discrete systems is the Roesser model (RM) that has been originally introduced in Roesser (1975). It is also possible to define the continuous or hybrid versions of Roesser model to describe $n$-D systems such as the following form (Bochniak and Gałkowski 2005)

$$
\mathbf{E}\left[\begin{array}{c}
\frac{\partial}{\partial t_{1}} x^{1}\left(t_{1}, \ldots, t_{r}, j_{r+1}, \ldots, j_{n}\right) \\
\vdots \\
\frac{\partial}{\partial t_{r}} x^{r}\left(t_{1}, \ldots, t_{r}, j_{r+1}, \ldots, j_{n}\right) \\
x^{r+1}\left(t_{1}, \ldots, t_{r}, j_{r+1}+1, \ldots, j_{n}\right) \\
\vdots \\
x^{n}\left(t_{1}, \ldots, t_{r}, j_{r+1}, \ldots, j_{n}+1\right)
\end{array}\right]=\mathbf{A}\left[\begin{array}{c}
x^{1}\left(t_{1}, \ldots, t_{r}, j_{r+1}, \ldots, j_{n}\right) \\
\vdots \\
\frac{x^{r}\left(t_{1}, \ldots, t_{r}, j_{r+1}, \ldots, j_{n}\right)}{x^{r+1}\left(t_{1}, \ldots, t_{r}, j_{r+1}, \ldots, j_{n}\right)} \\
\vdots \\
x^{n}\left(t_{1}, \ldots, t_{r}, j_{r+1}, \ldots, j_{n}\right)
\end{array}\right]
$$

The vectors $x^{h}\left(t_{1}, \ldots, t_{r}, j_{r+1}, \ldots, j_{n}\right) \in \mathbb{R}^{N_{h}}, h=1, . ., n$, where $\sum_{h=1}^{n} N_{h}=N$, are the local state subvectors. The global state vector is obtained by concatenating in a column all these subvectors. $\mathbf{A} \in \mathbb{R}^{N \times N}$ is a dynamic matrix, $\mathbf{E} \in \mathbb{R}^{N \times N}$ is introduced to consider implicit dynamics on this sequence. Notice that the hybrid formulation of positive 2D models has been recently considered by Kaczorek in Kaczorek (2000), also with fractional aspects (Kaczorek 2008, 2011).

Also note that in the classic formulation of Roesser models (Roesser 1975; Bochniak and Gałkowski 2005), the matrix $\mathbf{E}$ is the identity matrix. In the present note, we just slightly extend this formulation to implicit (or descriptor if prefered) systems. Our purpose is not to address the special issues encountered when dealing with implicit dynamics but simply to propose a framework for the derivation of LMI stability condition, which is as general as possible. Implicit models can be quite difficult to handle in the general $n \mathrm{D}$ context (Gałkowski 2000) but they can reasonably be considered when studying, for instance, iterative learning control (ILC) schemes, or repetitive processes (Rogers et al. 2007). Indeed, if the "along the pass dynamics" involve implicit dynamics, this implicit description will necessary make a matrix $\mathbf{E}$ appear in the corresponding Roesser model. The reader must also be aware that implicit does not necessarily mean singular. In other words, $\mathbf{E}$ is not necessarily rank deficient. In the sequel, for the sake of simplicity, matrix $\mathbf{E}$ is assumed non singular but if further investigation were to be led on the singular case, a particular attention should be paid to Kaczorek (1988a,b, 1990, 1992), Gałkowski (2000).

Moreover, matrices $\mathbf{A}$ and $\mathbf{E}$ are here assumed to be uncertain. More precisely, they are subject to parametric uncertainties in their entries. The parameters, denoted by $\delta_{i}$, 
$i=1, \ldots, p$, can be stacked into one single parameter vector $\delta$ which is assumed to belong to a set $\nabla_{\delta}$ defined by

$$
\nabla_{\delta}=\left\{\delta=\left[\begin{array}{c}
\delta_{1} \\
\vdots \\
\delta_{p}
\end{array}\right] \in \mathbb{R}^{p}: \underline{\delta} \leq \delta_{i} \leq \bar{\delta}_{i},\left[\begin{array}{c}
\underline{\delta}_{i} \\
\bar{\delta}_{i}
\end{array}\right] \in \mathbb{R}^{2} \forall i\right\},
$$

meaning that every real parameter belongs to a given finite range. Besides, in order to be quite general in the description of the uncertainty, the parameters are assumed to appear in rational expressions so we assume that $\mathbf{A}$ and $\mathbf{E}$ are described through implicit Linear Fractional Representations with respect to $\delta$ as follows:

$$
\left\{\begin{array}{l}
\mathbf{A}=\Delta_{A}(\delta) \star A \\
\mathbf{E}=\Delta_{E}(\delta) \star E
\end{array}\right.
$$

In the above expression, the matrix $\Delta$ is the "uncertainty matrix" defined by

$$
\left\{\begin{array}{l}
\Delta_{A}(\delta)=\bigoplus_{i=1}^{p} \delta_{i} I_{\mu_{i}} \in \mathbb{R}^{\mu \times \mu}, \\
\Delta_{E}(\delta)=\bigoplus_{i=1}^{p} \delta_{i} I_{\nu_{i}} \in \mathbb{R}^{v \times v},
\end{array}\right.
$$

with, of course, $\sum_{i=1}^{p} \mu_{i}=\mu$ and $\sum_{i=1}^{p} v_{i}=v$, and the matrices $A$ and $E$ are defined by

$$
[A \mid E]=\left[\begin{array}{cc|cc}
A_{A} & B_{A} & A_{E} & B_{E} \\
C_{A} & D_{A} & C_{E} & D_{E} \\
E_{A} & F_{A} & E_{E} & F_{E}
\end{array}\right]
$$

Moreover, the above LFR are assumed to be well-posed i.e. $\left(E_{A}-\Delta_{A}(\delta) A_{A}\right)^{-1}$ and $\left(E_{E}-\Delta_{E}(\delta) A_{E}\right)^{-1}$ exist over $\nabla_{\delta}$.

This so-called generalized (or implicit) LFR is a slight generalization of that proposed in Hecker and Varga (2004), Peaucelle et al. (2007). It must be noticed that it is possible to introduce different uncertain parameters in $\mathbf{A}$ and $\mathbf{E}$ but it is useless and this description is more natural since some parameters can appear in both $\mathbf{A}$ and $\mathbf{E}$.

Any reader familiar with LFRs-based uncertainties knows that a crucial issue can be the size of the square matrix $\Delta(\delta)$. There are infinitely many possible LFR which describe the same matrices $\mathbf{A}$ and $\mathbf{E}$ but it matters to find an LFR with a minimum size of matrix $\Delta(\delta)$. This is a well-known hard problem. Even if the system model is non descriptor, meaning that $\mathbf{E}=I_{N}$, it can be of high interest to consider implicit LFR on $\mathbf{A}$ (rather than a simple LFR where $E_{A}=I, F_{A}=0$ ). Indeed, they really can lead to a lower size of $\Delta_{A}(\delta)$ and $\Delta_{E}(\delta)$ (Hecker and Varga 2004; Manceaux-Cumer and Chrétien 2001; Sari et al. 2011). Note however that when the size of $\Delta_{A}(\delta)$ and $\Delta_{E}(\delta)$ increases, the conservatism does not always drastically increases (see the example in Sect. 5).

With such a general uncertain $n \mathrm{D}$ models at hand, we now aim at deriving, in a systematic fashion, robust stability conditions which are tractable from a computational point of view.

\section{LMI relaxation of stability conditions}

In this section, we first remind the reader of a stability condition which is here applied to model (2). 
Theorem 1 (Extension of Agathoklis 1988; Jury 1978; Piekarski 1977; Bochniak and Gałkowski 2005) Let the vector $\lambda$ and the matrix $\Delta_{\lambda}(\lambda)$ be defined by $\lambda=\left[\lambda_{1} \ldots \lambda_{n}\right]^{\prime}$ and

$$
\Delta_{\lambda}(\lambda)=\bigoplus_{h=1}^{n} \lambda_{h} I_{N_{h}} \in \mathbb{C}^{N}
$$

The system (2) is robustly asymptotically stable if and only if

$$
\operatorname{det}\left(\mathbf{E} \Delta_{\lambda}(\lambda)-\mathbf{A}\right) \neq 0, \quad \forall(\delta, \lambda) \in \nabla_{\delta} \times \nabla_{\lambda}
$$

where the set $\nabla_{\lambda}$ is defined by

$$
\nabla_{\lambda}=\left\{\lambda=\left[\begin{array}{c}
\lambda_{1} \\
\vdots \\
\lambda_{n}
\end{array}\right] \in \mathbb{C}^{k}:\left\{\begin{array}{l}
\operatorname{Re}\left(\lambda_{h}\right) \geq 0, h=1, \ldots, r, \\
\left|\lambda_{h}\right| \geq 1, h=r+1, \ldots, n
\end{array}\right\} .\right.
$$

Proof By rewriting the determinant in (8), the difference is equivalent to

$$
\operatorname{det}(\mathbf{E}) \operatorname{det}\left(\Delta_{\lambda}(\lambda)-\mathbf{E}^{-1} \mathbf{A}\right) \neq 0, \quad \forall(\delta, \lambda) \in \nabla_{\delta} \times \nabla_{\lambda}
$$

The reader is reminded of the nonsingularity of $\mathbf{E}$ (implicit nonsingular case). Thus, $\mathbf{E}^{-1}$ exists and $\operatorname{det}(\mathbf{E}) \neq 0$. Therefore, it comes

$$
\operatorname{det}\left(\Delta_{\lambda}(\lambda)-\mathbf{E}^{-1} \mathbf{A}\right) \neq 0, \quad \forall(\delta, \lambda) \in \nabla_{\delta} \times \nabla_{\lambda} .
$$

and the condition of Bochniak and Gałkowski (2005) is recovered with $\mathbf{E}^{-1} \mathbf{A}$ as a dynamic matrix.

Notice that this condition is not tractable from a computational point of view and concerns only stability but no other properties (regularity, impulse freeness or causality) which may be studied while dealing with singular descriptor models. Hence our assumption that $\mathbf{E}$ is nonsingular. However, even when $\mathbf{E}$ is singular, the present reasoning about stability is still valid. But some additional constraints have to be included in order to cope with the previoulsy mentioned properties, which goes beyond the topic of this article. Once again, see Kaczorek (1988a,b, 1990) for pioneering insights into the singular case.

From condition (8), we will now derive a sufficient LMI condition for robust stability.

The first step consists in using the properties given in appendix 7 to write $\left(\mathbf{E} \Delta_{\lambda}(\lambda)-\mathbf{A}\right)$ as an implict LFR. Using (50), the product $\mathbf{E} \Delta_{\lambda}(\lambda)$ can be written

$$
\mathbf{E} \Delta_{\lambda}(\lambda)=\left[\begin{array}{cc}
\Delta_{E}(\delta) & 0 \\
0 & \Delta_{\lambda}(\lambda)
\end{array}\right] \star\left[\begin{array}{cc|c}
A_{E} & B_{E} & 0 \\
0 & 0 & I \\
\hline C_{E} & D_{E} & 0 \\
\hline E_{E} & F_{E} & 0 \\
0 & I & 0
\end{array}\right] .
$$

Then, using the appendix, more precisely (49), the sum $(\mathbf{E} \Delta(\lambda)-\mathbf{A})$ complies with

$$
-\mathbf{A}+\mathbf{E} \Delta_{\lambda}(\lambda)=\left[\begin{array}{ccc}
\Delta_{A}(\delta) & 0 & 0 \\
0 & \Delta_{E}(\delta) & 0 \\
0 & 0 & \Delta_{\lambda}(\lambda)
\end{array}\right] \star\left[\begin{array}{cc}
\overline{\mathscr{A}} & \overline{\mathscr{B}} \\
\mathscr{C} & \mathscr{D} \\
\overline{\mathscr{E}} & \overline{\mathscr{F}}
\end{array}\right],
$$


with

$$
\left[\begin{array}{cc}
\overline{\mathscr{A}} & \overline{\mathscr{B}} \\
\mathscr{C} & \mathscr{D} \\
\overline{\mathscr{E}} & \overline{\mathscr{F}}
\end{array}\right]=\left[\begin{array}{ccc|c}
A_{A} & 0 & 0 & B_{A} \\
0 & A_{E} & B_{E} & 0 \\
0 & 0 & 0 & I \\
\hline-C_{A} & C_{E} & D_{E} & -D_{A} \\
\hline E_{A} & 0 & 0 & F_{A} \\
0 & E_{E} & F_{E} & 0 \\
0 & 0 & I & 0
\end{array}\right]
$$

Condition (8) can then be written

$$
\operatorname{det}\left(\Delta \star\left[\begin{array}{c|c}
\mathscr{A} & \mathscr{B} \\
\hline \mathscr{C} & \mathscr{D} \\
\hline \mathscr{E} & \mathscr{F}
\end{array}\right]\right) \neq 0, \quad \forall(\delta, \lambda) \in \nabla_{\delta} \times \nabla_{\lambda},
$$

where

$$
\Delta=\left[\begin{array}{cc}
\Delta(\delta) & 0 \\
0 & \Delta_{\lambda}(\lambda)
\end{array}\right] \text {, with } \Delta(\delta)=\underset{i=1}{\oplus} \delta_{i} I_{\mu_{i}+v_{i}},
$$

by re-arranging the rows of $\overline{\mathscr{A}}, \overline{\mathscr{B}}, \overline{\mathscr{E}}$, and $\overline{\mathscr{F}}$ to obtain $\mathscr{A}, \mathscr{B}, \mathscr{E}$ and $\mathscr{F}$ as in appendix 7 .

Define the following various values related to the parameter ranges:

$$
c_{i}=\frac{\underline{\delta}_{i}+\bar{\delta}_{i}}{2}, \quad g_{i}=\left(\frac{\bar{\delta}_{i}-\underline{\delta}_{i}}{2}\right)^{2}-c_{i}^{2}, \quad i=1, \ldots, p .
$$

Also define the set $\nabla$ as follows:

$$
\nabla=\left\{\Delta \in \mathbb{C}^{(\mu+v+N) \times(\mu+v+N)}:\left[\frac{\Delta}{I}\right]^{\prime} X\left[\frac{\Delta}{I}\right] \geq 0, \quad \forall X \in \mathbb{X}\right\}
$$

where $\mathbb{X}$ is a set of multipliers defined by

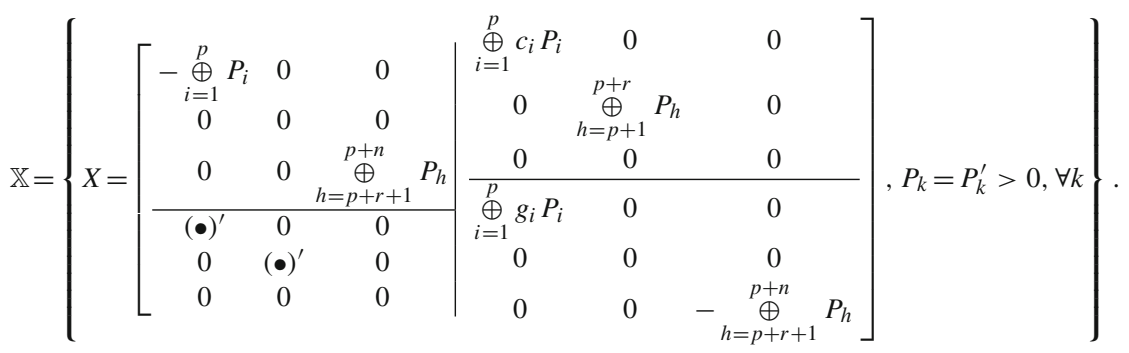

With such a definition of $\nabla$, it can be easily seen that

$$
(\delta, \lambda) \in \nabla_{\delta} \times \nabla_{\lambda} \Rightarrow \Delta \in \nabla .
$$

Unfortunately (we will come back to this issue), the reverse is not true. Therefore, $\nabla$ characterizes an overset of $\nabla_{\delta} \times \nabla_{\lambda}$. Nevertheless, condition (15) can be written

$$
\operatorname{det}\left(\mathscr{D}+\mathscr{C}(\mathscr{E}-\Delta \mathscr{A})^{-1}(\Delta \mathscr{B}-\mathscr{F})\right) \neq 0, \quad \forall(\delta, \lambda) \in \nabla_{\delta} \times \nabla_{\lambda}
$$

Due to (20), this condition holds if (but not only if)

$$
\operatorname{det}\left(\mathscr{D}+\mathscr{C}(\mathscr{E}-\Delta \mathscr{A})^{-1}(\Delta \mathscr{B}-\mathscr{F})\right) \neq 0, \quad \forall \Delta \in \nabla
$$




$$
\Leftrightarrow(\bullet)^{\prime} \underbrace{(\bullet)^{\prime}(-I)\left[\begin{array}{l}
\mathscr{C}^{\prime} \\
\mathscr{D}^{\prime}
\end{array}\right]^{\prime}}_{\Theta}\left[\begin{array}{c}
(\mathscr{E}-\Delta \mathscr{A})^{-1}(\Delta \mathscr{B}-\mathscr{F}) \\
I
\end{array}\right]<0, \quad \forall \Delta \in \nabla .
$$

By virtue of Corollary 1, this last inequality is equivalent to

$$
\exists X \in \mathbb{X}: \Theta+\left[\begin{array}{cc}
\mathscr{E} & \mathscr{F} \\
\mathscr{A} & \mathscr{B}
\end{array}\right]^{\prime} X\left[\begin{array}{cc}
\mathscr{E} & \mathscr{F} \\
\mathscr{A} & \mathscr{B}
\end{array}\right]<0 .
$$

The previous reasoning is summarized in the next theorem:

Theorem 2 The system (2) is robustly asymptotically stable if there exist Hermitian positive matrices $P_{k}=P_{k}^{\prime}>0, k=1, \ldots, \mu+v+n$ such that (24) holds with $\Theta$ and $X$ defined in (23) and (19) respectively.

Notice that this inequality is an LMI in the various matrices $P_{k}$ and thus is very tractable from a computational point of view.

\section{Discussion}

In this section, we propose comments about the meaning and the potentiality of Theorem 2. We particularly insist on the resemblance between the problems induced by parametric uncertainty and multidimensionality.

\subsection{Dimensionality, number of parameters and conservatism}

If one pays a little attention to condition (24), one can see that the decision variables in the LMI are all Hermitian matrices (possibly symmetric in practice as it is assumed in the sequel)

$$
\begin{cases}P_{i}=P_{i}^{\prime}>0, P_{i} \in \mathbb{R}^{\left(\mu_{i}+v_{i}\right) \times\left(\mu_{i}+v_{i}\right)} & \forall i \in\{1, \ldots, p\}, \\ P_{h}=P_{h}^{\prime}>0, P_{h} \in \mathbb{R}^{N_{h} \times N_{h}} & \forall h \in\{p+1, \ldots, n\} .\end{cases}
$$

So each parameter is considered in the LMI (as well as in matrix $\Delta$ ) in the same fashion as a Laplace or advance variable associated with a dimension, or the other way around. The main conservatism of the condition is highlighted in (20). This equation means that the property is ensured for any matrix $\Delta$ belonging to set $\nabla$ which is largest than the required set. Indeed, if one looks at the definition of $\nabla$ in (18), it is clear that there is no constraint that would force matrix $\Delta$ to comply with the structure given in (16). In other words, the LMI (24), when satisfied, guarantees that $\operatorname{det}\left(\mathbf{E} \Delta_{\lambda}(\lambda)-\mathbf{A}\right) \neq 0$ for the matrices $\Delta$ in $\nabla$ among which those of interest can be found. If $\nabla$ exactly described the concerned set, the S-procedure would be said lossless for our problem. Unfortunately, it is not the case; The structure given in (16) is not taken into account.

Roughly speaking, the conservatism increases with the number of zero entries in $\Delta$ as defined in (16). Consequently, the larger $p$ and $n$ are, the more conservative the LMI (24) is. (Moreover, it matters to exhibit LFR so as to reduce $\mu_{i}$ and $v_{i}$ as much as possible in order to also reduce the conservatism as well as the computation time.) It is not the first time that uncertain parameters are interpreted as new dimensions. This idea is also evoked in a completely different way in Rafajlowicz and Rafajlowicz (2011).

Several LMI or Lyapunov-like nominal stability conditions of $n \mathrm{D}$ systems encountered in the literature could be deduced with our approach. We here propose few classic examples of such LMI conditions. Indeed, consider a nominal purely continuous non implicit Roesser 
system i.e. $\mathbf{A}$ and $\mathbf{E}$ reduce to $D_{A}$ and $I$ respectively and $r=n$. Then the expression $\left(\Delta_{\lambda}(\lambda)-D_{A}\right)$ can be written

$$
\Delta_{\lambda}(\lambda)-D_{A}=\Delta_{\lambda}(\lambda) \star\left[\begin{array}{cc}
0 & I \\
I & 0 \\
I & D_{A}
\end{array}\right] .
$$

Following the same reasoning as in the previous section (but with much simpler expressions), a sufficient LMI condition for nominal stability is

$$
\exists X:\left[\begin{array}{cc}
-I & 0 \\
0 & 0
\end{array}\right]+\left[\begin{array}{cc}
I & D_{A} \\
0 & I
\end{array}\right]^{\prime} X\left[\begin{array}{cc}
I & D_{A} \\
0 & I
\end{array}\right]<0,
$$

with

$$
X=\left[\begin{array}{ll}
0 & P \\
P & 0
\end{array}\right], \quad P=\bigoplus_{h=1}^{n} P_{h}, \quad P_{h}=P_{h}^{\prime}>0,
$$

which, by Finsler's lemma (Finsler 1937), is equivalent to

$$
\exists X:\left[\begin{array}{c}
D_{A} \\
I
\end{array}\right]^{\prime} X\left[\begin{array}{c}
D_{A} \\
I
\end{array}\right]<0 .
$$

This inequality also writes

$$
D_{A}^{\prime} P+P D_{A}<0,
$$

which simply means that $\sum_{h=1}^{n} x^{h^{\prime}} P_{h} x^{h}$ is a Lyapunov function in the continuous sense for the system.

In the same fashion, consider a nominal purely discrete non implicit Roesser system i.e. $\mathbf{A}$ and $\mathbf{E}$ reduce to $D_{A}$ and $I$ and $r=0$, then the reasoning is the same except that, in this case, $X$ is defined by

$$
X=\left[\begin{array}{cc}
P & 0 \\
0 & -P
\end{array}\right], \quad \text { with } P=\bigoplus_{h=1}^{n} P_{h}, \quad P_{h}=P_{h}^{\prime}>0,
$$

which leads to

$$
-P+D_{A}^{\prime} P D_{A}<0
$$

meaning, once again, that $\sum_{h=1}^{n} x^{h^{\prime}} P_{h} x^{h}$ is a Lyapunov function in the discrete sense for the system.

If now, one comes back to a conventional 1D system, inequalities (30) and (32) are the usual Lyapunov's and Stein's inequalities respectively, in which $P=P^{\prime}$ is a full block positive definite matrix.

In such $1 \mathrm{D}$ cases, the $\mathrm{S}$-procedure is lossless so the conditions are not conservative. A little more general example can be considered. Let the following nominal 2D hybrid Roesser model be given:

$$
\left[\begin{array}{c}
\frac{\partial}{\partial t} x^{1}(t, j) \\
x^{2}(t, j+1)
\end{array}\right]=\underbrace{\left[\begin{array}{ll}
A_{11} & A_{12} \\
A_{21} & A_{22}
\end{array}\right]}_{D_{A}}\left[\begin{array}{l}
x^{1}(t, j) \\
x^{2}(t, j)
\end{array}\right] .
$$


Then, in that case, the structure of $X$ is as follows:

$$
X=\left[\begin{array}{cc|cc}
0 & 0 & P_{1} & 0 \\
0 & P_{2} & 0 & 0 \\
P_{1} & 0 & 0 & 0 \\
0 & 0 & 0 & -P 2
\end{array}\right], \quad P_{1}=P_{1}^{\prime}>0, \quad P_{2}=P_{2}^{\prime}>0
$$

If one makes the choice

$$
\Delta_{\lambda}(\lambda)-D_{A}=\Delta_{\lambda}(\lambda) \star\left[\begin{array}{cc}
0 & I \\
I & -D_{A} \\
I & 0
\end{array}\right]
$$

Then, still the same reasonning leads to the next LMI condition

$$
\left[\begin{array}{cc}
A_{21}^{\prime} P_{2} A_{21}+A_{11}^{\prime} P+P A_{11} & A_{21}^{\prime} P_{2} A_{22}+P_{1} A_{12} \\
A_{22}^{\prime} P_{2} A_{21}+A_{12}^{\prime} P_{1} & A_{22}^{\prime} P_{2} A_{22}-P_{2}
\end{array}\right]<0,
$$

in which $P_{1}$ acts as a Lyapunov matrix for the continuous dimension while $P_{2}$ acts as a Lyapunov matrix for the discrete dimension. If the choice (26) is made instead of (35), LMI (36) is also obtained.

But of course, these are only very special instances of the present contribution which enables large extensions to much more sophisticated analytical problems (see the next subsection).

Concerning the robustness aspects, there are of course many LMI results which cannot be considered as special cases of our approach because the considered uncertainties are not the same (norm-bounded case) or, when possible, are simply reformulated another way (polytopic case as in $\mathrm{Xu}$ et al. 2010). However, we claim that the present approach is very general and makes clearly the conservatism and the resemblance between the number of parameters and the dimensionality appear. Moreover, concerning the popular polytopic description of the parametric, the reader must be aware that if $\mathbf{A}$ and $\mathbf{E}$ do not depend linearly on the parameters $\delta_{i}$, then the polytopic description cannot be used a priori.

Coming back to the conservatism, another source of sufficiency comes from the fact that in the definition of $\nabla$, the realness of the parameters $\delta_{i}$ is not taken into account. Indeed the approach impicitely assume that each $\delta_{i}$ belongs to a disc of center $c_{i}$ with radius $\left(\frac{\bar{\delta}_{i}-\underline{\delta}_{i}}{2}\right)$ whereas it also belongs to the real axis. This realness can be taken into account, following the idea proposed in Iwasaki and Hara (2005), Scorletti Dinh et al. (2005), Bachelier and Mehdi (2006), by changing the structure of $X$ into

$$
X=X^{\prime}=\left[\begin{array}{ll}
X_{11} & X_{12} \\
X_{21} & X_{22}
\end{array}\right]
$$

with

$$
\left[\frac{X_{11}}{X_{21}}\right]=\left[\begin{array}{ccc}
-\oplus_{i=1}^{p} P_{i} & 0 & 0 \\
0 & 0 & 0 \\
0 & 0 & \oplus_{h=p+r+1}^{p+n} \\
& & P_{h} \\
(\bullet)^{\prime} & 0 & 0 \\
0 & (\bullet)^{\prime} & 0 \\
0 & 0 & 0
\end{array}\right] \text { and }
$$




$$
\left[\frac{X_{12}}{X_{22}}\right]=\left[\begin{array}{ccc}
\oplus_{i=1}^{p} c_{i} P_{i}-G_{j} & 0 & 0 \\
0 & \underset{\substack{p+r \\
h=p+1}}{\oplus} P_{h} & 0 \\
0 & 0 & 0 \\
\hline \bigoplus_{i=1}^{p} g_{i} P_{i} & 0 & 0 \\
0 & 0 & 0 \\
0 & 0 & \substack{p+n \\
h=p+r+1}
\end{array}\right],
$$

where $P_{k}=P_{k}^{\prime}>0$ and $G_{i}=-G_{i}^{\prime}$. This modification preserves the linearity with respect to the decision variables and thus just simply makes the LMI system a little more complicated, slighlty increasing the computation. The examples show that the reduction of conservatism, when it exists, is usually quite weak.

\subsection{About possible extensions}

To avoid even tougher notations, we have restricted our presentation to robust stability problems. Actually, the performances to be analyzed could be much more general. For instance, positive realness or bounded realness (i.e. $H_{\infty}$-level) of a system could be studied in a similar way. Indeed, Such properties can actually be expressed as in (56) with an appropriate choice of $\Theta$ (different from that considered in (23)) and thus tested through S-procedure. In fact, these properties can be connected to the so-called Kalman-Yakubovich-Popov (KYP) lemma (Willems 1971; Rantzer 1996) a $n$ D version of which has been proposed in Bachelier et al. (2008). This would correspond to an extension of the results of Bachelier et al. (2008) to the case where the matrices are subject to uncertain parameters as introduced in Sect. 2. This could have been done here but perhaps it would have led to undue complications which would have blurred the message we want to convey.

Going on with perspectives, the design is of course an interesting challenge. Many LMI analytical conditions have been extended to design, especially the state feedback control law. This is also something we are about to address. It seems that this approach is more powerful for analysis than for synthesis, at least at first sight.

\section{An illustrative example}

To illustrate the relevance of the present contribution, the next model can be considered:

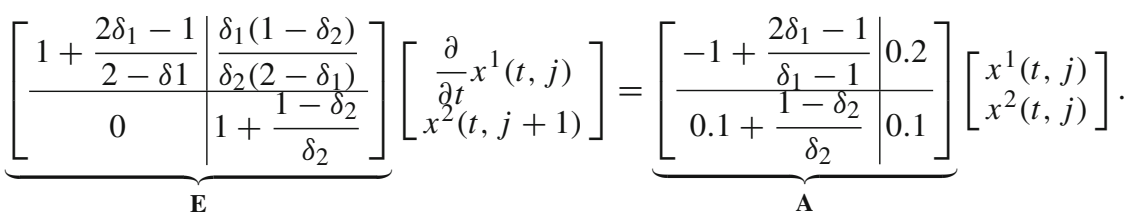

This system is a 2D hybrid Roesser model for which $N=n=2$ and which involves two uncertain parameters $\delta_{1}$ and $\delta_{2}$ in a rational way. These parameters are assumed to satisfy

$$
\left\{\begin{array}{l}
\underline{\delta_{1}}=\delta_{1_{0}}(1-\alpha) \leq \delta_{1} \leq \bar{\delta}_{1}=\delta_{1_{0}}(1+\alpha), \\
\underline{\delta_{2}}=\delta_{2_{0}}(1-\alpha) \leq \delta_{2} \leq \bar{\delta}_{2}=\delta_{2_{0}}(1+\alpha),
\end{array}\right.
$$


where $\delta_{1_{0}}=0.5$ and $\delta_{2_{0}}=1$ are the nominal values of the uncertain parameters and where $\alpha>0$ is some sort of size of the box $\nabla_{\delta}$ where $\delta$ lies.

Matrices $A$ and $E$ can be formulated as implict LFRs as follows:

$$
A=\left[\begin{array}{cc}
\delta_{1} & 0 \\
0 & \delta_{2}
\end{array}\right] \star\left[\begin{array}{rr|rr}
1 & 0 & 2 & 0 \\
0 & 1 & 1 & 0 \\
\hline-1 & 0 & -1 & 0.2 \\
0 & 1 & 0.1 & 0.1 \\
\hline 1 & 0 & 1 & 0 \\
0 & 0 & 1 & 0
\end{array}\right]
$$

and

$$
E=\left[\begin{array}{cc}
\delta_{1} & 0 \\
0 & \delta_{2}
\end{array}\right] \star\left[\begin{array}{ll|ll}
1 & 1 & 2 & 0 \\
0 & 1 & 0 & 1 \\
\hline 1 & 0 & 1 & 0 \\
0 & 1 & 0 & 1 \\
\hline 2 & 0 & 1 & 0 \\
0 & 0 & 0 & 1
\end{array}\right]
$$

With the notations in Eqs. (14) and (15), it comes

$$
\begin{aligned}
{\left[\begin{array}{rr}
\overline{\mathscr{A}} & \overline{\mathscr{B}} \\
\mathscr{C} & \mathscr{D} \\
\overline{\mathscr{E}} & \overline{\mathscr{F}}
\end{array}\right] } & =\left[\begin{array}{rrrrrr|rr}
1 & 0 & 0 & 0 & 0 & 0 & 2 & 0 \\
0 & 1 & 0 & 0 & 0 & 0 & 1 & 0 \\
0 & 0 & 1 & 1 & 2 & 0 & 0 & 0 \\
0 & 0 & 0 & 1 & 0 & 1 & 0 & 0 \\
0 & 0 & 0 & 0 & 0 & 0 & 1 & 0 \\
0 & 0 & 0 & 0 & 0 & 0 & 0 & 1 \\
\hline 1 & 0 & 1 & 0 & 1 & 0 & 1 & -0.2 \\
0 & -1 & 0 & 1 & 0 & 1 & -0.1 & -0.1 \\
\hline 1 & 0 & 0 & 0 & 0 & 0 & 1 & 0 \\
0 & 0 & 0 & 0 & 0 & 0 & 1 & 0 \\
0 & 0 & 2 & 0 & 1 & 0 & 0 & 0 \\
0 & 0 & 0 & 0 & 0 & 1 & 0 & 0 \\
0 & 0 & 0 & 0 & 1 & 0 & 0 & 0 \\
0 & 0 & 0 & 0 & 0 & 1 & 0 & 0
\end{array}\right] \\
\qquad\left[\begin{array}{rrrrrr|rr}
1 & 0 & 0 & 0 & 0 & 0 & 2 & 0 \\
\mathscr{A} & \mathscr{B} \\
\mathscr{C} & \mathscr{D} \\
\mathscr{E} & \mathscr{F}
\end{array}\right] & =\left[\begin{array}{rrrrrrrr}
0 \\
0 & 1 & 0 & 0 & 0 & 0 & 1 & 0 \\
0 & 0 & 0 & 1 & 0 & 1 & 0 & 0 \\
0 & 0 & 0 & 0 & 0 & 0 & 1 & 0 \\
0 & 0 & 0 & 0 & 0 & 0 & 0 & 1 \\
\hline 1 & 0 & 1 & 0 & 1 & 0 & 1 & -0.2 \\
0 & -1 & 0 & 1 & 0 & 1 & -0.1 & -0.1 \\
\hline 1 & 0 & 0 & 0 & 0 & 0 & 1 & 0 \\
0 & 0 & 2 & 0 & 1 & 0 & 0 & 0 \\
0 & 0 & 0 & 0 & 0 & 0 & 1 & 0 \\
0 & 0 & 0 & 0 & 0 & 1 & 0 & 0 \\
0 & 0 & 0 & 0 & 1 & 0 & 0 & 0 \\
0 & 0 & 0 & 0 & 0 & 1 & 0 & 0
\end{array}\right] .
\end{aligned}
$$


Matrix $\Delta$ has the following structure:

$$
\Delta=\left[\begin{array}{cccc}
\delta_{1} I_{2} & 0 & 0 & 0 \\
0 & \delta_{2} I_{2} & 0 & 0 \\
0 & 0 & \lambda_{1} & 0 \\
0 & 0 & 0 & \lambda_{2}
\end{array}\right] \in \mathbb{C}^{6 \times 6}
$$

To test robust stability, the considered structure of the multilplier $X$ is the one given by (19) with

$$
\begin{aligned}
& c_{1}=\delta_{i_{0}}, \quad g_{i}=\delta_{i_{0}}\left(\alpha^{2}-1\right), \quad \text { and } P_{i}=P_{i}^{\prime} \in \mathbb{R}^{2 \times 2}, \quad \forall i \in\{1 ; 2\}, \\
& P_{i}=P_{i} \in \mathbb{R}, \quad \forall i \in\{3 ; 4\},
\end{aligned}
$$

and with

$$
p=2, r=1, n=2 .
$$

Notice that $P_{1}$ and $P_{2}$ are associated with $\delta_{1}$ and $\delta_{2}$ while $P_{3}$ and $P_{4}$ are associated with $\lambda_{1}$ and $\lambda_{2}$, i.e. with the continuous and discrete dynamics which are of course coupled.

By applying Theorem 2, i.e. by testing the feasibility of LMI condition (24), solutions are found for all $\alpha$ such that

$$
\alpha \in[0 ; 0.68775] \text {. }
$$

( $\alpha=0$ corresponds to the nominal case.) Beyond this upper value 0.68775 , the LMI is found infeasible meaning that the stability cannot be proved owing to this condition (but it may still hold since the condition is conservative).

As an example, for $\alpha=0.5$, the solutions proposed by the solver are

$$
\begin{aligned}
& P_{1}=\left[\begin{array}{ll}
1.2526 & 0.5714 \\
0.5714 & 0.6914
\end{array}\right]>0, \\
& P_{2}=\left[\begin{array}{rr}
0.3547 & -0.3236 \\
-0.3236 & 0.5238
\end{array}\right]>0, \\
& P_{3}=0.2068, \quad P_{4}=0.1416 .
\end{aligned}
$$

For another choice of LFRs which is far from optimal $\left(\Delta \in \mathbb{R}^{10 \times 10}\right)$, the greatest value of $\alpha$ for which stability could be assessed is 0.68766 (the details are omitted since the LFR is very cumbersome). Thus, this is just slightly lower than with the small LFR since the best value of $\alpha$ has been reduced by only $0.013 \%$ and the question of numeric accuracy is of course raised. The computation time is just a bit greater (less than twice). However, this is just an example and small LFRs are easier to handle from many points of view.

\section{Conclusion}

In this paper, we have proposed a quite generic approach to analyse the robust stability of implicit hybrid Roesser models. The uncertain parameters appear through implicit LFR in the entries of the system matrices so the description is very general. The obtained conditions are expressed in terms of LMI, so they are very tractable from a computational point of view. Extensions to a larger class of problems beyond stability, e.g. positive realness or bounded realness should soon follow. The design of control laws might be more difficult. 


\section{A Some useful properties of implicit LFR}

In this appendix, we focus a little on the properties of implicit LFRs. We only give the three properties that are useful in the body of the paper.

Let three implicit LFRs be given:

$$
\begin{gathered}
M_{1}=\Delta_{1} \star\left[\begin{array}{ll}
A_{1} & B_{1} \\
C_{1} & D_{1} \\
E_{1} & F_{1}
\end{array}\right], \\
M_{2}=\Delta_{2} \star\left[\begin{array}{ll}
A_{2} & B_{2} \\
C_{2} & D_{2} \\
E_{2} & F_{2}
\end{array}\right] . \\
M=\bar{\Delta} \star\left[\begin{array}{cc}
\bar{A} & \bar{B} \\
C & D \\
\bar{E} & \bar{F}
\end{array}\right],
\end{gathered}
$$

with $\bar{\Delta}$ a block diagonal matrix which satisfies

$$
\bar{\Delta}=T^{-1} \Delta T
$$

where $\Delta$ is also a block diagonal matrix and where $T$ is a matrix which enables the permuation of the blocks in $\Delta$ to get $\vec{\Delta}$. Then it comes:

Summation:

$$
M_{1}+M_{2}=\left[\begin{array}{cc}
\Delta_{1} & 0 \\
0 & \Delta_{2}
\end{array}\right] \star\left[\begin{array}{cc|c}
A_{1} & 0 & B_{1} \\
0 & A_{2} & B_{2} \\
\hline C_{1} & C_{2} & D_{1}+D_{2} \\
\hline E_{1} & 0 & F_{1} \\
0 & E_{2} & F_{2}
\end{array}\right]
$$

Multiplication:

$$
M_{1} M_{2}=\left[\begin{array}{cc}
\Delta_{1} & 0 \\
0 & \Delta_{2}
\end{array}\right] \star\left[\begin{array}{cc|c}
A_{1} & B_{1} C_{2} & B_{1} D_{2} \\
0 & A_{2} & B_{2} \\
\hline C_{1} & D_{1} C_{2} & D_{1} D_{2} \\
\hline E_{1} & F_{1} C_{2} & F_{1} D_{2} \\
0 & E_{2} & F_{2}
\end{array}\right]
$$

Block permutation:

$$
M=\Delta \star\left[\begin{array}{cc}
A & B \\
C & D \\
E & F
\end{array}\right]
$$

with

$$
\left[\begin{array}{ll}
A & B \\
E & F
\end{array}\right]=\left[\begin{array}{ll}
T & 0 \\
0 & T
\end{array}\right]\left[\begin{array}{cc}
\bar{A} & \bar{B} \\
\bar{E} & \bar{F}
\end{array}\right]
$$




\section{Descriptor concrete S-procedure}

In this appendix, we first recall the abstract full block S-procedure (Yakubovich 1971) formulated about in the same way as in Scherer (2001) and then we make it more concrete by specializing it to the case where implicit LFRs are involved.

Theorem 3 (Scherer 2001) Let the following mathematical objects be introduced:

- $\nabla$, a compact set of complex matrices $\Delta$;

- An Hermitian matrix $\Theta$;

- A matrix $V \in \mathbb{R}^{l \times n}$;

- $\mathscr{S}(\Delta)$, a family of subspaces $\mathbb{C}^{l}$ continuously depending on $\Delta$ over $\nabla$;

- $\mathscr{B}(\Delta)=\left\{x \in \mathbb{C}^{n}: V x \in \mathscr{S}(\Delta)\right\}, \Delta \in \nabla$.

Then the next two statements are equivalent :

a)

$$
x^{\prime} \Theta x<0 \quad \forall x \in \mathscr{B}(\Delta) \backslash\{0\}, \quad \forall \Delta \in \nabla .
$$

b)

$$
\exists X:\left\{\begin{array}{l}
V^{\prime} X V+\Theta<0 \\
z^{\prime} X z \geq 0 \quad \forall z \in \mathscr{S}(\Delta), \forall \Delta \in \nabla
\end{array}\right.
$$

Corollary 1 Let $\mathbb{X}$ be set of multipliers $X$ which enables the characaterization of a compact set of matrices $\Delta$ defined as follows:

$$
\nabla=\left\{\Delta:\left[\begin{array}{c}
\Delta \\
I
\end{array}\right]^{\prime} X\left[\begin{array}{c}
\Delta \\
I
\end{array}\right] \geq 0, \forall X \in \mathbb{X} .\right\}
$$

Then the two following statements are equivalent:

i) $\forall \Delta \in \nabla$,

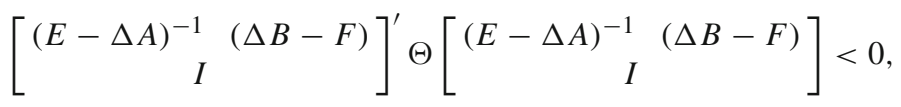

ii)

$$
\exists X \in \mathbb{X}:\left[\begin{array}{ll}
E & F \\
A & B
\end{array}\right]^{\prime} X\left[\begin{array}{cc}
E & F \\
A & B
\end{array}\right]+\Theta<0
$$

Proof This is an application of Theorem 3. Indeed Assume that the subspace $\mathscr{S}(\Delta)$ is defined as

$$
\mathscr{S}(\Delta)=\operatorname{Ker}([I-\Delta]),
$$

where $\operatorname{Ker}($.$) denotes the right nullspace of a matrix. The elements of \mathscr{S}(\Delta)$ can be characterized by

$$
z=\left[\begin{array}{c}
\Delta \\
I
\end{array}\right] q, \quad q \in \mathbb{C}^{2 \mu}
$$


Therefore, with such a characterization, the definition of $\nabla$ in (55) is such that it makes the second inequality in (54) verified. Indeed, it becomes the definition of $\nabla$ itself. Moreover, the matrix $V$ is chosen as follows:

$$
V=\left[\begin{array}{ll}
E & F \\
A & B
\end{array}\right]
$$

Then for any vector $x$ of the form

$$
x=\left[\begin{array}{rr}
(E-\Delta A)^{-1} & (\Delta B-F) \\
I &
\end{array}\right],
$$

ones gets

$$
[I-\Delta] V x=0,
$$

which proves that $V x$ belongs to $\mathscr{S}(\Delta)$ i.e. that $x$ belongs to $\mathscr{B}(\Delta)$ as defined in Theorem 3 . Thus, applying Theorem 3 amounts to proving the equivalence between statements i) and ii).

\section{References}

Agathoklis, P. (1988). Lower bounds for the stability margin of discrete two-dimensional systems based on the two-dimensional Lyapunov equation. IEEE Transactions on Circuits and Systems, 35(6), 745-749.

Bachelier, O., \& Mehdi, D. (2006). Robust matrix root-clustering through extended KYP lemma. SIAM Journal of Control and Optimization, 45(1), 368-381.

Bachelier, O., Paszke, W., \& Mehdi, D. (2008). On the Kalman-Yakubovih-Popov lemma and the multidimensional models. Multidimensional Systems and Signal Processing, 19(3-4), 425-447.

Bochniak, J., \& Gałkowski, K. (2005). LMI-based analysis for continuous-discrete linear shift invariant nDsystems. Journal of Circuits, Systems and Computers, 14(2), 1-26.

Bose, N. K. (1982). Applied multidimensional systems theory. New York: Van Nostrand-Reinhold.

Boyd, S., El Ghaoui, L., Feron, E., \& Balakrishnan, V. (1994). Linear matrix inequalities in system and control theory, volume 15 of SIAM studies in applied and numerical mathematics. Philadelphia: SIAM.

Bracewell, R. N. (1995). Two-dimensional Imaging. Prentice Hall Signal Processing Series. Upper Saddle River: Prentice Hall Inc.

Dinh, M., Scorletti, G., Fromion, V., \& Magarotto, E. (2005). Parameter dependent $\mathrm{H}_{\infty}$ control by finite dimensional LMI optimization: Application to trade-off dependent control. International Journal of Robust and Nonlinear Control, 15, 383-406.

Dudgeon, D. E., \& Merserau, R. M. (1984). Multidimensional digital signal processing. Englewood Cliffs: Prentice-Hall Signal Processing Series. Prentice Hall.

Ebihara, Y., Ito, Y., \& Hagiwara, T. (2006). Exact stability analysis of 2-D systems using LMIs. IEEE Transactions on Automatic Control, 51(9), 1509-1513.

Finsler, P. (1937). Über das Vorkommen definiter und semidefiniter Formen in Scharen quadratischer Formen: Comment. Mathematici Helvetici, 9, 188-192.

Fornasini, E., \& Marchesini, G. (1978). Doubly indexed dynamical systems: State models and structural properties. Mathematical Systems Theory, 12, 59-72.

Gałkowski, K. (2000). A perspective on singularity in 2D linear systems. Multidimensional Systems and Signal Processing, 11(1-2), 83-108.

Gałkowski, K., Lam, J., Xu, S., \& Lin, Z. (2003). LMI approach to state-feedback stabilization of multidimensional systems. International Journal of Control, 76(14), 1428-1436.

Gałkowski, K., \& Wood, J. (Eds.). (2001). Multidimensional signals, circuits and systems. Systems and control book series. London: Taylor and Francis.

Hecker, S., \& Varga, A. (2004). Generalized LFT-based representation of parametric models. European Journal of Control, 10(4), 326-337.

Henrion, D., Sebek, M., \& Bachelier, O. (2001). Rank-one LMI approach to stability of 2-D polynomial matrices. Multidimensional Systems and Signal Processing, 12(1), 33-48. 
Hinamoto, T. (1993). 2-D Lyapunov equation and filter design based on the Fornasini-Marchesini second model. IEEE Transactions on Circuits and Systems I: Fundamental Theory and Applications, 40(2), 102110.

Iwasaki, T., \& Hara, S. (2005). Generalized KYP lemma: unified frequency domain inequalities with design applications. IEEE Transactions on Automatic Control, 50(1), 41-59.

Jury, E. (1978). Stability of multidimensional scalar and matrix polynomial. Proceedings of the IEEE, 66(9), 1018-1047.

Kaczorek, T. (1985). Two-dimensional linear systems, volume 68 of Lecture Notes in Control and Information Sciences. Berlin: Springer.

Kaczorek, T. (1988a). The singular general model of 2-D systems and its solution. IEEE Transactions on Automatic Control, 33(11), 1060-1061.

Kaczorek, T. (1988b). Singular multidimensional linear discrete systems. In Proceedings of IEEE international symposium on circuits and systems. Helsinki, Finland, June 1988b, pp. 105-108.

Kaczorek, T. (1990). General response formula and minimum energy control for the general singular model of 2-d systems. IEEE Transactions on Automatic Control, 35(4), 433-436.

Kaczorek, T. (1992). Linear control systems, vol. 2. New York: Research Studies Press, Wiley.

Kaczorek, T. (2000). Positive 1D and 2D systems. Communications and control engineering. London: Springer.

Kaczorek, T. (2008). Positive fractional 2D hybrid linear systems. Bulletin of the Polish Academy of SciencesTechnical Sciences, 56(3), 273-277.

Kaczorek, T. (2009). LMI approach to stability of 2d positive systems. Multidimensional Systems and Signal Processing, 20(1), 39-54.

Kaczorek, T. (2011). Selected problems of fractional systems theory. Berlin: Springer.

Lu, W.-S. (1994). On a Lyapunov approach to stability analysis of 2-D digital filters. IEEE Transactions on Circuits and Systems I: Fundamental Theory and Applications, 41(10), 665-669.

Lu, W.-S., \& Antoniou, A. (1992). Two-dimensional digital filters, vol. 80 of electrical engineering and elecronics. New York: Marcel Dekker, Inc.

Manceaux-Cumer, C., \& Chrétien, J.-P. (2001). Minimal LFT form of a spacecraft built up from two bodies. In AIAA Guidance, navigation, and control conference. Quebec, Canada, , pp. 105-108, August 2001.

Peaucelle, D., Arzelier, D., Henrion, D., \& Gouaisbault, F. (2007). Quadratic separation for feedback connection of an uncertain matrix and an implicit linear transformation. Automatica, 43, 796-804.

Piekarski, M. (1977). In Algebraic characterization of matrices whose multivariable characteristic polynomial is Hurwitzian. In Proceedings of international symposium Operator Theory. Lubbock, USA.

Rafajlowicz, E., \& Rafajlowicz, W. (2011). $(n+r)$ D systems and their control with reduced sensitivity to parametric uncertainties. In Proceedings of 7th international workshop on multidimensional systems (nDS'2011). Poitiers, France.

Rantzer, A. (1996). On the Kalman-Yakubovich-Popov lemma. Systems and Control Letters, 28(1), 7-10.

Roesser, R. P. (1975). A discrete state-space model for linear image processing. IEEE Transactions on Automatic Control, 20(1), 1-10.

Rogers, E., Gałkowski, K., \& Owens, D. H. (2007). Control systems theory and applications for linear repetitive processes, vol. 349 of Lecture Notes in Control and Information Sciences. Berlin: Springer.

Sari, B., Bachelier, O., \& Mehdi, D. (2011). Robust S-regularity of matrix pencils applied to the analysis of descriptor models. Linear Algebra and its Applications, 435(5), 923-942.

Scherer, C. W. (2001). LPV control and full block multipliers. Automatica, 37, 361-375.

Shi, Y. Q., \& Zhang, X. M. (2002). A new two-dimensional interleaving technique using successive packing. IEEE Transactions on Circuits and Systems I: Fundamental Theory and Applications, 49(6), 779-789.

Willems, J. C. (1971). Least squares stationary optimal control and the algebraic Riccati equation. IEEE Transactions on Automatic Control, 16(6), 621-634.

$\mathrm{Xu}, \mathrm{H}$., Lin, Z., \& Makur, A. (2010). Non-fragile $\mathrm{H}_{2}$ and $\mathrm{H}_{\infty}$ filter designs for polytopic two-dimensional systems in Roesser model. Multidimensional Systems and Signal Processing, 31(3), 255-275.

Yakubovich, V. A. (1971). S-procedure in nonlinear control theory. Vestnik Leningrad University, 1, 62-77. 


\section{Author Biographies}
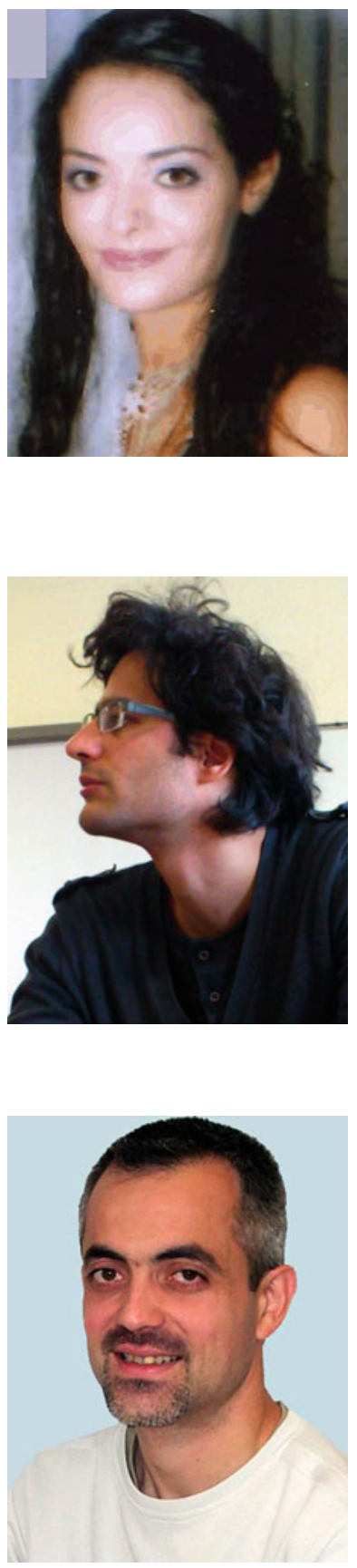

Mariem Ghamgui was born in Sfax, Tunisia, in 1985. She received her engineering degree from Sfax University, Tunisia, in 2009. She is actually $\mathrm{PhD}$ student in the LIAS (Laboratoire d'Informatique et d'Automatique pour les Systèmes) which depends on the ENSIP (École Nationale Supérieure d'Ingénieurs de Poitiers) and on the ENSMA (École Nationale Supérieure de Mécanique et d'Aérotechnique) in Poitiers. Her areas of interest include two dimensional systems and time delay systems.

Nima Yeganefar received his PhD from the École Centrale de Lille and the University of Lille in 2006. He joined the University of Poitiers as a Lecturer in 2009 in the LIAS laboratory (Laboratoire d'Informatique et d'Automatique pour les Systèmes). His area of interest includes Lyapunov stability, multidimensional systems, and timedelay systems. On his spare time, he loves having friendly chat with his colleagues about GMOs and other hot scientific topics.

Olivier Bachelier was born in Châteauroux, France, in 1971. He received the engineer's degree and the masters' degree with specialization in control from the INSA (Institut National des Sciences Appliquées), Toulouse, France, in 1994 and then the Ph.D. degree, still from the INSA, in 1998. In 1999, he joined the IUT (Institut Universitaire de Technologie) of Poitiers-Châtellerault-Niort and the LIAS (Laboratoire d'Informatique et d'Automatique pour les Systèmes) which depends on the ENSIP (École Nationale Supérieure d'Ingénieurs de Poitiers) and on the ENSMA (École Nationale Supérieure de Mécanique et d'Aérotechnique) in Poitiers, where he has been working up to now. He received the habilitation in 2009 and was appointed professor in 2012, still in the IUT. (The IUT and the ENSIP are parts of the University of Poitiers). Olivier Bachelier is studying robust analysis and robust design of uncertain multi-input multi-ouput linear models. $\mathrm{He}$ is particularly interested in robust matrix root-clustering, pole and eigenstructure assignment, descriptor systems and multidimensional models. 


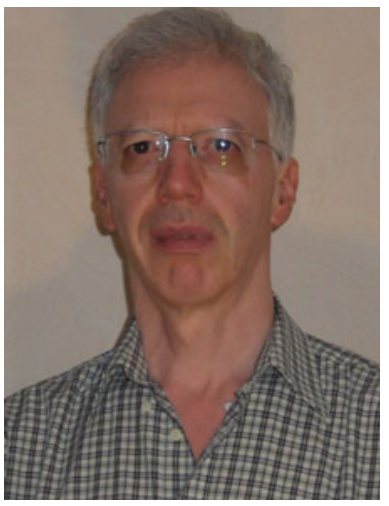

Driss Mehdi received an engineer degree from Mohammadia Engineering school, Rabat, Morocco in 1979 and a $\mathrm{PhD}$ degree in Automatic control from Nancy University in 1986. He was Senior Lecturer from 1988 to 1992 at the Louis Pasteur University of Strasbourg, France, and from 1992 he has been working as a Professor at the University of Poitiers. His main interests are Automatic control, Robust control, Delay and Descriptor systems. 